http://jmscr.igmpublication.org/home/ ISSN (e)-2347-176x ISSN (p) 2455-0450 crossref DOI: https://dx.doi.org/10.18535/jmscr/v7i8.154

Journal Of Medical Science And Clinical Research

\title{
Scientific study on bacteriological profile of neonatal sepsis in tertiary care hospital
}

Authors

\author{
Dr Nagalekshmi, Dr Ramesh Kumar
}

Department of Paediatrics, Kanyakumari Government Medical College, Asaripallam

\begin{abstract}
Introduction: Neonatal sepsis is the most common problem encountered in all neonatal intensive care unit. It is a significant cause of morbidity and mortality in developing countries like India. Therefore neonatal sepsis needs early and accurate diagnosis and management with appropriate antibiotics for better outcome among NICU graduates

This study was aimed to analyze the clinical profile, bacterial isolates in culture positive neonates and bacteriological sensitivity pattern of microbial organisms

Material and Method: All culture positive neonatal cases admitted in our NICU KKGMCH from January 2018 - December 2018 were analyzed retrospectively

Study Design: Retrospective Descriptive study

Results: Total of 90 cultures positive neonatal cases were analyzed. Among them about 35 neonates were preterm which constitutes about $36 \%$. Microbial organism most common in our nicu is klebsiella which constitutes around 39\% and staphylococcus about 31\%, coagulase negative staphylocoicci constitutes 9\%, pseudomonas 9\%. Klebsiella is sensitive to cefotaxime, piperacillin and tazobactum and ciprofloxacin, staphylococcus organism is sensitive to clindamycin and linezolid pseudomonas sensitive to cefaperazone sulbactum and piperacillin.

Keywords: neonatal sepsis, bacterial isolates, bacteriological sensitivity.
\end{abstract}

\section{Introduction}

Neonatal sepsis is a clinical syndrome characterized by signs and symptoms of infection with or without accompanying bacteremia in the first month of life. There are varied presentation such as pneumonia, septicemia, meningitis, osteomyelitis , arthritis. ${ }^{(1)}$

Neonatal sepsis is the most common cause of neonatal morbidity and mortality. It constitutes about $50 \%$ of newborn death in nicu in developing countries like India. The incidence of neonatal sepsis is around 30 per1000 live birth based on National Neonatal and Perinatology Data ${ }^{(2)}$. There is higher incidence of sepsis in term male babies than female babies ${ }^{(3)}$

Neonatal sepsis may be categorized as early onset (day of life $0-3$ ) or late onset (day of life 4 or later). Of newborns with early-onset sepsis, $85 \%$ present within 24 hours (median age of onset 6 hours), $5 \%$ present at $24-48$ hours, and a smaller percentage present within $48-72$ hours. ${ }^{(2)}$ Onset is most rapid in premature neonates.

\section{Etiology}

The most commonest organisms are gram _-ve organisms like E,coli, klebsiella pseudomonas. 
staphylococcus aureus, coagulase negative staphylococcus $^{(4)}$

Early-onset sepsis is associated with acquisition of microorganisms from the mother. The risk factors for EOS are preterm labor, prolonged rupture of membrane, foul smelling liquor, multiple vaginal examination and maternal fever. EOS commonly manifests as pneumonia. Infection can occur via hematogenous, transplacental spread from an infected mother or more commonly, via ascending infection from the cervix. LOS infections are usually acquired from external environment. $^{(2)}$

Hypothermia is the most common manifestation of sepsis. Term babies manifests with fever ,poor suck, respiratory distress, diarrhea, abdominal distension, apnoeic spells, hepatitis, hepatosplenomegaly, high pitched cry, convulsions, bulging fontanel, sclerema, shock and DIC. ${ }^{(4)}$

Sepsis screening includes total leukocyte count, absolute neutrophil count, immature or band cells, micro ESR, C- reactive protein, csf analysis in suspected case of meningitis. The culture studies are confirmatory test

\section{Aim of the Study}

This study aims to analyze the bacteriological and clinical profile in culture positive sepsis cases admitted in NICU KKGMCH

\section{Materials and Methods}

This is a retrospective descriptive study in culture positive cases admitted in NICU. Details of sex, gestational age, birth weight, c- reactive protein, organisms detected in blood culture and their antibiotic sensitivity data were collected. The babies were further subdivided based on onset of symptoms as EOS and LOS

\section{Results}

A total of 90 culture positive neonates were analyzed. Among them 55 were male and 35 were females babies. On analyzing the gestation there were 56 term babies and 34 preterm babies.
Klebsiella is the most common organism detected in blood cultures in our nicu which constitutes around $39 \%$ and staphylococcus about $31 \%$ coagulase negative staphylocoicci constitutes $9 \%$ pseudomonas 9\%. Majority of the klebsiella positive blood cultures were sensitive to cefotaxime, piperacillin and tazobactum and ciprofloxacin. Majority of the staphylococcus organisms were sensitive to clindamycin and linezolid and majority of the pseudomonas sensitive to cefaperazone sulbactum and pipercillin. EOS was more common in our neonates than LOS. On analyzing the comorbidities birth asphyxia and TTNB were significant

\section{Discussion}

Among the 90 neonates, 55 were male babies and 35 female babies

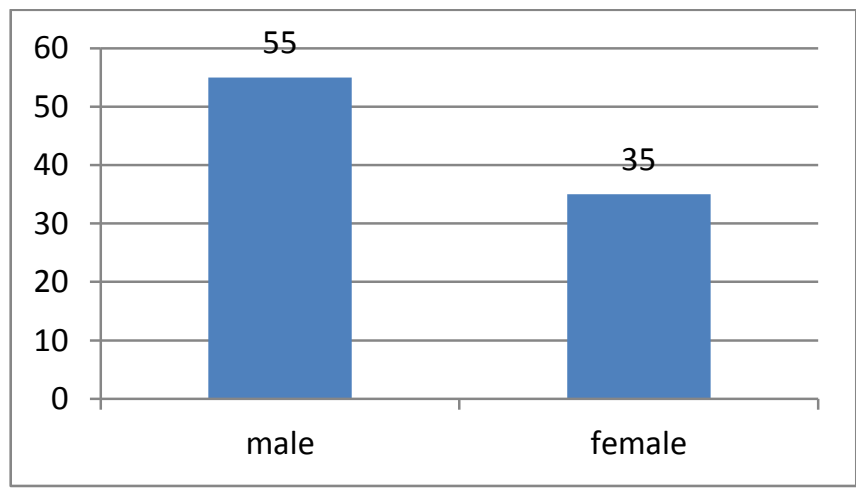

Figure 1: Distribution according to Gender

Among the total of 90 neonates analyzed 56 were term and 34 were preterm babies.

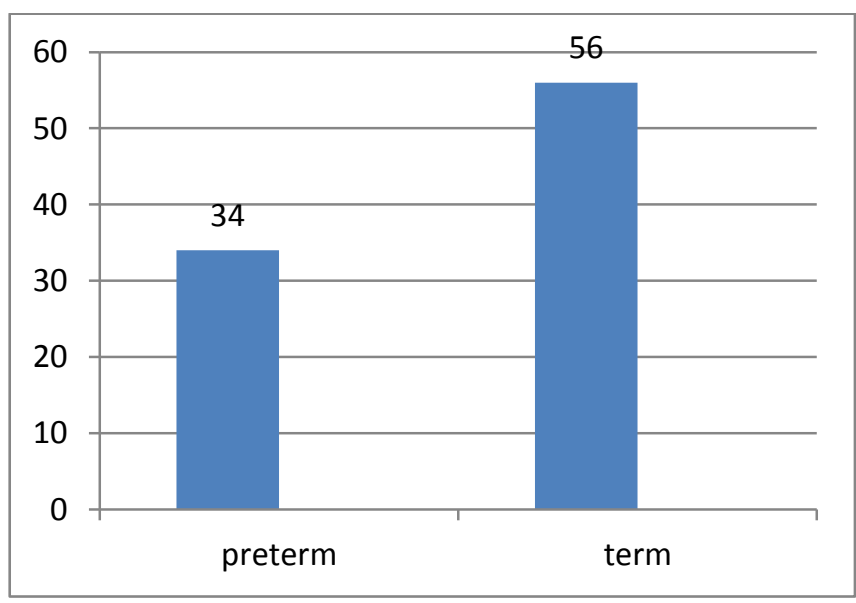

Figure 2: Distribution according to Gestational Age 
EOS is more prevalent in our nicu compared to LOS. There were 62 cases of EOS and 28 cases of LOS

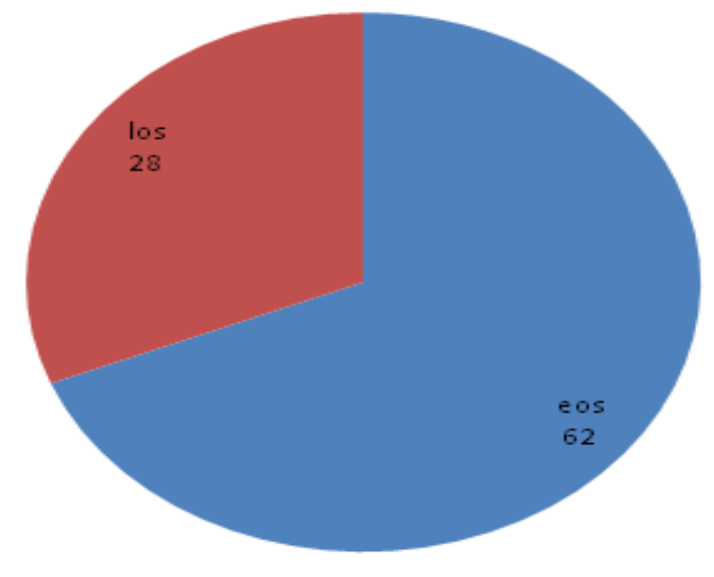

Figure 3: Distribution based On classification of sepsis

Microbial organism which is most common in our nicu is klebsiella which constitutes around 39\% and staphylococcus about $31 \%$ coagulase negative staphylococci constitutes $9 \%$ pseudomonas $9 \%$

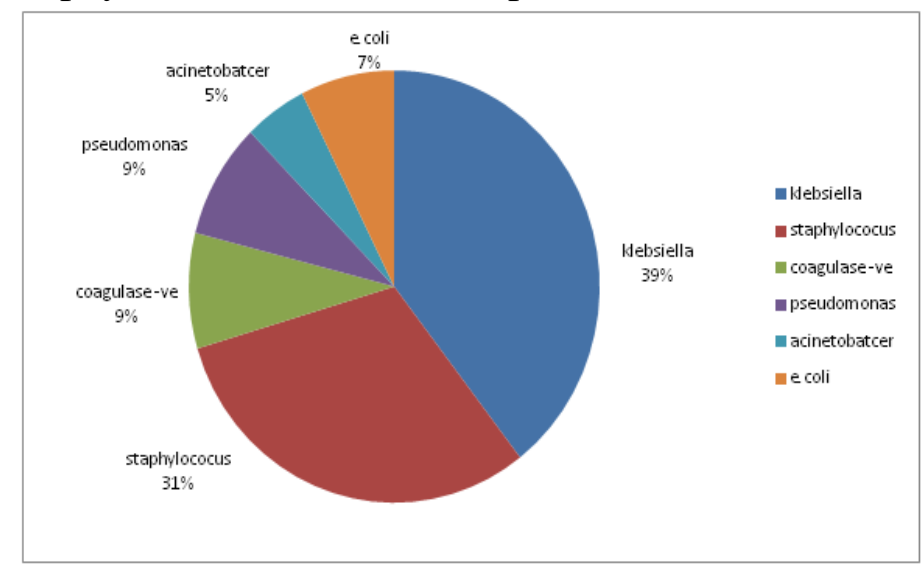

Figure 4: Distribution of prevalence of various organisms

Acinetobacter is about 5\% and $\mathrm{E}$.coli constitutes about 7\%. On analyzing the organisms most prevalent among EOS neonates is klebsiella and in LOS neonates staphylococcus is more prevalent. Majority of the klebsiella positive blood cultures were sensitive to cefotaxime, piperacillin and tazobactum and ciprofloxacin. Majority of the staphylococcus organisms were sensitive to clindamycin and linezolid and majority of the pseudomonas is sensitive to cefaperazonesulbactum and piperacillin. On analyzing the data on drug resistance of the organisms the gram negative organisms are resistant to amoxycillin clavulanic acid, ampicillin, gentamycin, cefixime and cefepime and gram +ve organisms are resistant to penicillin and erythromycin.

On analyzing the clinical profile pneumonia is the most common clinical manifestation in sepsis babies which constitutes about 53\% and meningitis constitutes about $5 \%$ and septic arthritis 5\% .Birth asphyxia is more common comorbidity in sepsis babies( $30 / 90)$.

Comparison with previous studies

In South India a study done by paven kumar et al showed EOS is more common which is similar to our study and staphylococcus was most prevalent in their nicu followed by klebsiella. ${ }^{(5)}$ In our study klebsiella is more common than staphylococcus. Another study conducted in bhuvaneswar by urvashi Rana et al showed the results similar to our study as klebsiella is more prevalent followed by staphylococcus and in resistance to organisms majority of isolates were resistant to ampicillin and gentamycin similar to our study ${ }^{(6)}$. Studies done in Nepal showed staphylococcus aureus and coagulase negative staphylococcus are the commonest organism but the pathogens showed sensitive to third generation cephalosporins and gentamycin and amikacin. ${ }^{(7)}$. Study on bacteriological profile of neonatal sepsis by madanjeet kumar from bhuvaneswar also showed results similar to our study in pathogens and were sensitive to amikacin and meropenem ${ }^{(8)}$. A study by sangita gupta from delhi on neonatal sepsis showed coagulase negative staphylococci and $\mathrm{k}$. pneumonia are the commonest isolates and most of the pathogens were multidrug resistant ${ }^{(9)}$

\section{Conclusion}

In our NICU gram negative organisms like klebsiella is most prevalent pathogen, followed by staphylococcus in culture positive neonates .The pathogens are resistant to ampicillin and gentamycin. So early screning of sepsis and 
appropriate choice of antibiotics is essential to prevent emergence of multidrug resistance and reserve antibiotics for future infants

\section{References}

1. AIIMS protocols in neonatalogy second edition

2. Care of the newborn meharban singh

3. Nelsons textbook of paediatrics edition 20

4. O.P Ghai textbook of paediatrics

5. Rashmi p, Praveen BK. Clinico bacteriological profile of neonatal sepsis. Int . J contemp Pediatr 2019:796-802

6. Urvashi rana clinicobacteriological profile of neonatal sepsis in a tertiary care ARC Iournal of pediatrics volume 2 issue 2 2016

7. Shrestha RK bacteriological study of neonatal sepsis and antibiotic sensitivity Nepal Med Coll . J 2013 March 15 (1)71 $-3$

8. Dr Madanjeet Kumar Study of bacteriological profile of neonatal sepsis in a tertiary care hospital prevalent microorganism and their susceptibility patterns IOSR Journal of dental and medical sciences

9. Gupta .S. Singh VK, Singhal S. Chowdhary P. Neonatal sepsis in a tertiary care hospital in Delhi, India: study of microbial profile and antimicrobial susceptibility pattern. Int .J Contemp Pediatr 2019:6. 